\title{
Coherent wavefield subtraction for diffraction separation
}

\author{
Benjamin Schwarz \\ University of Oxford, Department of Earth Sciences, \\ South Parks Road, Oxford OX1 3AN, United Kingdom \\ (January 7, 2019) \\ Running head: Coherent diffraction separation
}

\begin{abstract}
Diffractions encode sub-wavelength information and superior illumination but are weak in amplitude and strongly interfere with the more dominant reflected wavefield. Accordingly, the successful separation of diffractions in geophysical applications still forms a major direction of research. Confronting this challenge, an automatable and versatile framework for the separation of interfering wavefields through a sequence of coherent data summation and subtraction is proposed. In contrast to other studies utilizing coherence measurements, the method specifically targets reflected contributions, which are normally favored in automated summation schemes, and adaptively subtracts the resulting reflection stack from the input data. Complementing this natural selectivity of stacking, a variety of wavefront filters is presented, which can be derived from the local maximization of the semblance norm and additionally inform the subsequent separation step. Complex 2D and 3D synthetic preand poststack seismic examples together with a ground-penetrating radar (GPR) field data example suggest that the presented scheme can be applied to a variety of data configurations and bears the potential to uncover an extremely faint but surprisingly rich diffraction background that was previously not accessible for dedicated processing.
\end{abstract}




\section{INTRODUCTION}

For at least half a century, most of the advances in seismic exploration, in terms of employed methods and data acquisition, have been made for the reflected wavefield. In fact, even today, a majority of the day-to-day seismic processing is still mostly dominated by reflections and only recently, other wavefield components, including refractions observable through modern long-offset acquisitions, and diffractions have been gaining increasing interest. In particular in the context of localized structural complexity such as faults seismic diffraction provides a robust and highly resolved means of detection (e.g., Krey, 1952; Landa and Keydar, 1998; Khaidukov et al., 2004). In addition, diffraction has the unique property that it does not honor Snell's law and, thus, radiates uniformly, resulting in far superior illumination compared to reflections, which lets it appear very favorable e.g. for velocity inversion (Fomel et al., 2007; Reshef and Landa, 2009; Santos et al., 2012; Bauer et al., 2017). As a consequence, the multi-channel kinematic response of a point diffraction is fully encoded in data subsets, such as common-shot gathers or the zero-offset section (e.g., Bauer et al., 2016; Schwarz and Gajewski, 2017a). One - if not the major - reason why these unique properties of diffractions could not yet be fully harnessed in dedicated processing strategies is that, due to geometrical spreading, the diffracted energy is distributed over a significantly larger surface area and amplitudes recorded at individual receivers at the surface are, consequently, so weak they can barely be recognized with the human eye. In addition, due to the nature of back-scattering, reflected and diffracted wavefields often heavily interfere, which makes quantitative investigations into the diffraction's nature hard to accomplish in practice (Kozlov et al., 2004).

Because of this strong interference of reflection and diffraction, a range of methods 
has been introduced in recent years to effectively separate diffractions from the rest of the recorded wavefield. Kirchhoff migration, as one of the work horses in seismic exploration, has its roots in optics and diffraction, according to Huygen's principle, represents a natural ingredient (e.g., Born and Wolf, 2013). Following this notion, first powerful attempts at seismic diffraction imaging were concerned with systematically incorporating specularity constraints in the Kirchhoff integral (Moser and Howard, 2008). While this led to highly resolved images of discontinuities, it was also shown that the quality of the images is sensitive to the velocity model at hand. Following these extensions of the Kirchhoff integral, other migration methods have been shown to likewise being naturally capable of extracting and imaging diffractions, provided the macro-velocity model available is reasonably accurate (e.g., Dafni and Symes, 2017; Yin and Nakata, 2017).

Introducing more robustness, Klokov and Fomel (2012) have demonstrated that data transformations, such as specific versions of the Radon transform, in conjunction with the use of time migration velocities, can lead to an efficient diffraction separation and imaging without the knowledge of macro-velocities in depth. In addition, different varieties of plane-wave destruction filters were demonstrated to efficiently separate diffractions without the prerequisite of detailed velocity information (e.g., Fomel, 2002; Fomel et al., 2007; Merzlikin et al., 2017). Qualitative and quantitative investigations have shown that the diffracted wavefield can likewise be distinguished solely in the data (time) domain by performing local coherence measurements (Berkovitch et al., 2009). Building on this early work, Dell and Gajewski (2011) introduced a fully data-driven workflow for diffraction separation and imaging, which was later extended to three dimensions and the prestack domain (Bakhtiari Rad et al., 2018). By utilizing multi-dimensional stacking (e.g., Gelchinsky et al., 1999a,b; Jäger et al., 2001; Schwarz and Gajewski, 2017a), these approaches, in contrast to 
migration-based alternatives, do not aim at focusing the diffracted energy, but rather make it accessible and usable e.g. for velocity model building via wavefront tomography (Bauer et al., 2017). However, the way they are implemented, both these methods have in common that the quality of the separation heavily relies on the quality of the performed coherence measurements, which is known to suffer from strong interference of reflected and diffracted events (Mann, 2001; Müller, 2009; Walda and Gajewski, 2017).

Only very recently, following the mentality of adaptive surface-related multiple elimination (e.g., Verschuur et al., 1992; Guitton and Verschuur, 2004) and plane-wave destruction (Fomel, 2002), Schwarz and Gajewski (2017b) have introduced a strategy in which the reflected rather than the diffracted wavefield is specifically targeted and approximated through coherent summation and then adaptively subtracted from the input data. As a result and in contrast to e.g. Dell and Gajewski (2011) and Bakhtiari Rad et al. (2018), this approach makes use of the usually undesired directional filter characteristics of stacking and was shown, in conjunction with subsequent coherence analysis in the diffraction-only data, to be capable of extracting a very rich diffraction background that was almost entirely hidden by reflections. Complementing Schwarz and Gajewski (2017b), in this work, I revisit the aforementioned concept of coherent wavefield subtraction and systematically investigate its applicability in the pre- and poststack domain and for 3D seismic data. In addition, I give insight into aspects of implementation and demonstrate with a field data example of the US Geological Survey (USGS), that the presented framework is likewise naturally applicable to ground-penetrating radar (GPR) measurements.

\section{METHOD}

[Figure 1 about here.] 
My approach to diffraction separation builds on the assumption that diffuse, complicated wavefields can be thought of as being composed of simple ones (Born and Wolf, 2013). Even seemingly random noise, as it can e.g. be observed when seismically investigating crystalline rocks contains spatially and temporally coherent signals that can be used for imaging (see, e.g., Ahmed et al., 2015). In essence, the employed strategy can be divided into three constituent stages:

1. Coherent wavefield summation

2. Wavefront filter estimation

3. Coherent wavefield subtraction

In order for coherence to be detected and efficiently utilized, the emerging wavefield needs to be sampled densely - a requirement that is mostly met in controlled-source seismology as well as in ground-penetrating radar (GPR) measurement campaigns. The above sequence of coherent data summation and subtraction will be discussed in detail in the following three subsections and is illustrated in Figure 1.

\section{Coherent wavefield summation}

Owing to the maximal geometrical spreading, diffraction results in wavefields whose amplitudes decay rapidly with propagation distance (e.g. Born and Wolf, 2013). Consequently, diffractions are rarely observable on individual records and commonly masked by interfering more amplitude-strong contributions such as reflections. So aside from an overall low signalto-noise ratio, the suppression of interfering wavefields - without harming the diffractions themselves - constitutes one of the main challenges of diffraction imaging. From an inter- 
ference viewpoint, the recorded seismic data, parameterized by a $2 \mathrm{D}$ vector $\mathbf{x}_{0}$ indicating the respective trace's location within the acquisition surface and the recording time $t_{0}$, can be written as a superposition of $k$ coherent wavefields $\mathcal{C}_{k}\left(\mathbf{x}_{0}, t_{0}\right)$ and random noise $\mathcal{N}\left(\mathbf{x}_{0}, t_{0}\right)$,

$$
\mathcal{D}\left(\mathbf{x}_{0}, t_{0}\right)=\sum_{k} \mathcal{C}_{k}\left(\mathbf{x}_{0}, t_{0}\right)+\mathcal{N}\left(\mathbf{x}_{0}, t_{0}\right)
$$

Due to the averaging involved, the process of stacking was shown to be an efficient means of suppressing random noise (Mayne, 1962). In the recent past it was demonstrated that data summation can be carried out along multi-dimensional surfaces for zero-offset or finite-offset reference traces (Gelchinsky et al., 1999a,b; Jäger et al., 2001; Zhang et al., 2001), which among other things, opens the door to full-prestack data regularization and enhancement schemes (Höcht et al., 2009; Baykulov and Gajewski, 2009; Xie and Gajewski, 2017). As an unwanted side effect it was observed that, owing to its common implementation as an optimization problem, the most dominant coherent arrivals recorded at a particular data point are usually favored. While there exist various attempts of overcoming this so-called conflicting dip problem (Soleimani et al., 2009; Höcht et al., 2009; Walda and Gajewski, 2017), one of the locally interfering wavefields $\mathcal{C}_{k}$ can be approximated through coherent summation within a specified aperture around the central data point $\left(\mathbf{x}_{0}, t_{0}\right)$,

$$
\mathcal{C}_{k}\left(\mathbf{x}_{0}, t_{0}\right) \approx \frac{1}{n} \sum_{i=1}^{n} \mathcal{D}\left[\mathbf{x}_{0}+\Delta \mathbf{x}_{i}, t_{0}+\Delta t_{k}\left(\Delta \mathbf{x}_{i}\right)\right]
$$

The index $i$ denotes the individual trace considered and $n$ is the number of traces included. As before, the index $k$ identifies a specific data-space direction that is parameterized by $\Delta t_{k}$ - the traveltime moveout belonging to the considered locally coherent event. Consequently, with the knowledge of $\Delta t_{k}$ at the considered trace locations $\Delta \mathbf{x}_{i}$ (with respect to the central trace) the $k$-th event can be approximated. However, with the absence of a-priori knowledge about the subsurface velocity structure, the correct moveout is not known, but 
rather needs to be extracted from the data themselves (e.g. Taner and Koehler, 1969). For this extraction to be performed in a data-driven fashion, an objective measure of coherence needs to be introduced, of which the semblance norm arguably is one of the most robust and widely used representations (Neidell and Taner, 1971). Taking only values between 0 (for total incoherence) and 1 (perfect coherence), the search for the best-fitting moveout can be phrased as a fully automatable optimization problem,

$$
\max _{\Delta t_{k}} \frac{1}{n} \frac{\sum_{\delta t}\left\{\sum_{i=1}^{n} \mathcal{D}\left[\mathbf{x}_{0}+\Delta \mathbf{x}_{i}, t_{0}+\Delta t_{k}\left(\Delta \mathbf{x}_{i}\right)\right]\right\}^{2}}{\sum_{\delta t} \sum_{i=1}^{n} \mathcal{D}^{2}\left[\mathbf{x}_{0}+\Delta \mathbf{x}_{i}, t_{0}+\Delta t_{k}\left(\Delta \mathbf{x}_{i}\right)\right]}
$$

where $\delta t$ is a time window, in which vertical summation is performed. Commonly its size is chosen to be constant, in correspondence with the predominant signal period, but in principle, its length can be systematically altered with time to account e.g. for effects of attenuation. While many other coherence measures exist in literature (e.g. Fomel, 2009; Asgedom et al., 2012) the semblance norm maximized in expression (3) is a very intuitive and insightful quantity, as it represents the ratio of possible constructive interference of a wavefield with itself and the total energy contained in it. So aside from viewing the data as a superposition of interfering coherent contributions and noise, as expressed in equation (1), the coherence of a wavefield can be viewed as its potential to self-interfere. Based on recent experience (e.g. Barros et al., 2015; Walda and Gajewski, 2017), in all presented data examples, the coherence maximization was performed with a global differential evolution optimizer (Storn and Price, 1997).

\section{Wavefront filter estimation}

Due to the fact that amplitude-strong contributions are normally favored, the aforementioned optimization problem (3) itself, in conjunction with directional stacking according 
to (2), is already sufficient to arrive at an approximately diffraction-free dataset (Schwarz and Gajewski, 2017b) that can be subtracted from the input data (compare figures 2(a) and (b)). However, since diffraction can also be prominent, when strong impedance contrasts, as e.g. encountered in salt environments, are present, I suggest to support the directional filtering due to the stacking process with filter functions that are derived from wavefront attributes that parameterize the traveltime moveout $\Delta t_{k}$ estimated in the frame of the coherence maximization. When controlled-source seismic or GPR measurements are concerned, it is safe to assume that reflections are the recorded wavefield components that most strongly interfere with the medium's diffraction response. Accordingly, equation (1), in good approximation, simplifies to

$$
\mathcal{D}\left(\mathbf{x}_{0}, t_{0}\right) \approx \mathcal{C}_{\text {ref }}\left(\mathbf{x}_{0}, t_{0}\right)+\mathcal{C}_{\text {diff }}\left(\mathbf{x}_{0}, t_{0}\right)+\mathcal{N}\left(\mathbf{x}_{0}, t_{0}\right)
$$

where $\mathcal{C}_{\text {ref }}$ and $\mathcal{C}_{\text {diff }}$ denote the coherent reflected and diffracted contributions and $\left(\mathbf{x}_{0}, t_{0}\right)$ indicates a specific data point. In contrast to previous works, which aimed at steering the stacking operators towards the desired diffracted contributions, I make use of the fact that reflections are naturally favored and only provide assisting constraints to ensure that no diffracted energy is unintentionally removed in the final subtraction stage.

Since, according to equation (1), in the most general case, different coherent wavefields can intersect at $\left(\mathbf{x}_{0}, t_{0}\right)$, different moveout expressions are required to govern the data's full complexity. Conversely, the concept of traveltime moveout can be used to efficiently discriminate between interfering wavefields. While in conventional velocity analysis, traveltime moveout is parameterized with effective velocities, paraxial ray theory delivers moveout approximations that are expressed in terms of zeroth, first, and second-order wavefront attributes (e.g. Ursin, 1982; Hubral, 1983; Zhang et al., 2001). Most generally, a data point 
is spatially characterized by two $2 \mathrm{D}$ vectors $\Delta \mathrm{x}_{i}^{s}$ and $\Delta \mathrm{x}_{i}^{g}$ denoting the relative source and receiver distances with respect to the reference source and receiver positions, respectively. In its hyperbolic approximation, the 3D prestack traveltime moveout for the $k$-th coherent event reads (Schleicher et al., 1993)

$$
\begin{aligned}
{\left[t_{0}+\Delta t_{k}\left(\Delta \mathbf{x}_{i}^{s}, \Delta \mathbf{x}_{i}^{g}\right)\right]^{2} } & =\left(t_{0}+\mathbf{p}_{k}^{s} \Delta \mathbf{x}_{i}^{s}+\mathbf{p}_{k}^{g} \Delta \mathbf{x}_{i}^{g}\right)^{2} \\
& +t_{0}\left[\left(\Delta \mathbf{x}_{i}^{s}\right)^{T} \mathbf{M}_{k}^{s} \Delta \mathbf{x}_{i}^{s}+\left(\Delta \mathbf{x}_{i}^{g}\right)^{T} \mathbf{M}_{k}^{g} \Delta \mathbf{x}_{i}^{g}\right] \\
& +2 t_{0}\left(\Delta \mathbf{x}_{i}^{s}\right)^{T} \mathbf{M}_{k}^{s g} \Delta \mathbf{x}_{i}^{g}
\end{aligned}
$$

with $\mathbf{p}_{k}^{s}, \mathbf{p}_{k}^{g}, \mathbf{M}_{k}^{s}$, and $\mathbf{M}_{k}^{g}$ denoting the slope vectors and curvature matrices describing traveltime changes at the reference source and reference receiver, respectively. The mixed-order matrix $\mathbf{M}_{k}^{s g}$ encodes Snell's law as it couples the source and receiver moveout contributions and, thus, can be used for diffraction / reflection discrimination (e.g. Bauer et al., 2016; Schwarz and Gajewski, 2017a). Since for the conventional reflection case, data redundancy is most pronounced in relative midpoint $\left(\Delta \mathbf{x}_{i}^{m}\right)$ and half source-receiver offset $\left(\mathbf{h}_{i}\right)$ coordinates, the zero-offset version of moveout (5) has gained increased popularity and was efficiently implemented in the frame of the 3D common-reflection-surface (CRS) stack (Bergler et al., 2002). For the $k$-th coherent event it can be expressed as

$$
\begin{aligned}
{\left[t_{0}+\Delta t_{k}\left(\Delta \mathbf{x}_{i}^{m}, \mathbf{h}_{i}\right)\right]^{2} } & =\left(t_{0}+2 \mathbf{p}_{k} \Delta \mathbf{x}_{i}^{m}\right)^{2} \\
& +2 t_{0}\left[\left(\Delta \mathbf{x}_{i}^{m}\right)^{T} \mathbf{M}_{k} \Delta \mathbf{x}_{i}^{m}+\mathbf{h}_{i}^{T} \mathbf{H}_{k} \mathbf{h}_{i}\right]
\end{aligned}
$$

While in the more general finite-offset source-receiver formulation, the slope vectors and curvature matrices are more closely related to the physical experiment, they describe fictitious one-way equivalent wavefront propagation in the common-midpoint (CMP) gather and the zero-offset section (Hubral, 1983; Jäger et al., 2001; Zhang et al., 2001). If the wavefield is sufficiently well sampled, the first-order subsets of expressions (5) and (6), cor- 
responding to slant stacks in the pre- and poststack domain, may already be sufficient to perform the suggested method of diffraction separation via coherent wavefield subtraction. For a more detailed discussion of traveltime moveout in the wavefront picture, I refer to the works of Gelchinsky et al. (1999a,b), Jäger et al. (2001), or Schwarz and Gajewski (2017a).

Generally, the potential for efficient discrimination of diffraction and reflection crucially depends on the considered data configuration. As discussed by Schwarz and Gajewski (2017b) for single-channel seismic data, equation (5) (for $\Delta \mathbf{x}_{i}^{s}=\mathbf{0}$ or $\Delta \mathbf{x}_{i}^{g}=\mathbf{0}$ ) and equation (6) (for $\mathbf{h}_{i}=\mathbf{0}$ ) reduce to (twice) the transmission response of a buried passive seismic source in specific sub-domains. Consequently, sufficient thresholding of $\mathbf{p}_{k}$ and/or $\mathbf{M}_{k}$ can be used for diffraction discrimination in essentially all imaginable data configurations. The two functions

$$
\begin{aligned}
F\left(\mathbf{p}_{k}\right) & =1-\frac{1}{2} \sum_{u} \Theta\left(p_{u}-p_{0}\right), \\
F\left(\mathbf{M}_{k}\right) & =1-\frac{1}{4} \sum_{u} \sum_{v} \Theta\left(M_{u v}-M_{0}\right),
\end{aligned}
$$

in which $\Theta$ denotes the Heaviside step function and $p_{u}$ and $M_{u v}$ correspond to the measured components of $\mathbf{p}_{k}$ and $\mathbf{M}_{k}$, are discontinuous. They take the value 1 for $p_{u}<p_{0}$ and $M_{u v}<M_{0}$ for all rows $u$ and columns $v$ and 0 the other way around. Normally, reflections reveal lower slope and curvature values than diffractions. Therefore, the binary character of filter functions $F\left(\mathbf{p}_{k}\right)$ and $F\left(\mathbf{M}_{k}\right)$ can be used for reflection and diffraction identification provided reasonable threshold values $p_{0}$ and $M_{0}$ are chosen.

While the success of this strategy crucially depends on the choice of these thresholds, a comparison of different estimated attributes promises a more data-driven discrimination. If multi-channel data was acquired, a very efficient way of discriminating reflected and diffracted contributions was introduced by Dell and Gajewski (2011) who compared the 
event curvatures observed in the common-midpoint gather and the zero-offset section, respectively. Bakhtiari Rad et al. (2018) provide an extended 3D analog of this criterion for the case of $3 \mathrm{D}$ point diffraction. Both these works aimed at directly extracting the diffraction response and, therefore, suffer from the strong wavefield interference that can almost always be observed. Here, as indicated before, I rather aim at approximating and extracting the coherent reflected wavefield and adaptively subtract it from the input data. Reformulated for this objective, the function

$$
F\left(\mathbf{M}_{k}, \mathbf{H}_{k}\right)=1-\exp \left(-\frac{\sum_{u} \sum_{v}\left|M_{u v}-H_{u v}\right|}{\sum_{u} \sum_{v}\left|M_{u v}+H_{u v}\right|}\right)
$$

takes high values (close to 1) for the reflection case and vanishes, if diffractions are considered, where $\mathbf{M}_{k} \approx \mathbf{H}_{k}$. Like before, $u$ and $v$ indicate the individual rows and columns of the two curvature matrices. Due to the fact that diffraction events reveal most pronounced curvature near the apex where slope values are reasonably low, a joint use of both threshold criteria (7) for the single-channel case or a combination of (7a) and (8) for multi-channel acquisitions promises the best results. However, as will be demonstrated in the following, diffractions can also be fully preserved using (7a) only, provided reasonably large apertures and/or time windows are chosen in the subtraction stage. For this reason, exemplarily, I only made use of functions (7a) and (8) individually in the presented data examples.

\section{Coherent wavefield subtraction}

[Figure 2 about here.]

[Figure 3 about here.]

In contrast to previous works, and very much in the tradition of adaptive surface- 
related multiple elimination (Verschuur et al., 1992), the coherent subtraction strategy aims at sufficiently well approximating the interfering reflected wavefield and subsequently subtracting it from the input data. As was described in the previous subsections, this first approximation step can routinely be performed through coherent summation (compare (2)), i.e.

$$
\mathcal{C}_{\mathrm{ref}}\left(\mathbf{x}_{0}, t_{0}\right) \approx \frac{1}{n} \sum_{i=1}^{n} \mathcal{D}\left(\mathbf{x}_{i}, \mathbf{t}_{i}\right)
$$

with $\mathbf{x}_{i}=\mathbf{x}_{0}+\Delta \mathbf{x}_{i}$ and $\mathbf{t}_{i}=t_{0}+\Delta t_{\text {ref }}\left(\Delta \mathbf{x}_{i}\right)$. For the sake of simplicity, from now on, I parameterize the position of a data trace by a single 2-component vector $\mathbf{x}_{i}$. Please note, however, that the presented arguments likewise apply to the more general finite-offset multi-channel case, where a source and a receiver coordinate vector are given. The main foundation of the process of coherent subtraction constitutes in the assumption, that the recorded wavefield can be viewed as a superposition of individual coherent contributions and incoherent noise (see equation (1)). Specifically, when the interference of reflection and diffraction is predominant, as is mostly the case in seismic exploration, we can rewrite expression (4) as

$$
\mathcal{C}_{\text {diff }}\left(\mathbf{x}_{0}, t_{0}\right)+\mathcal{N}\left(\mathbf{x}_{0}, t_{0}\right)=\mathcal{D}\left(\mathbf{x}_{0}, t_{0}\right)-\mathcal{C}_{\text {ref }}\left(\mathbf{x}_{0}, t_{0}\right)
$$

So, in principle, a subtraction of (9) from the input data $\mathcal{D}$, according to the above equation, should lead to a seismic section that only contains diffracted energy and uncorrelated noise. However, due to local wavefield complexity and data imperfections, as well as the effect of spread-length bias, a simple subtraction is rarely successful and likely to suffer from residuals that can be an order of magnitude stronger in amplitude than the faint diffracted contributions. To overcome these limitations, I suggest to employ local amplitude scaling factors $\alpha_{0}$ and time shifts $\tau_{0}$ (defined at $\mathbf{x}_{0}$ for time $t_{0}$ ) to the coherent reflection stack $(9)$ 
(compare Figure 2(c) and (d)). Accordingly, the least-squares misfit between input data and adapted reflection stack reads

$$
\Psi\left(\alpha_{0}, \tau_{0}\right)=\sum_{\delta t} \sum_{i}\left[\mathcal{D}\left(\mathbf{x}_{i}, \mathbf{t}_{i}\right)-\alpha_{0} \mathcal{C}_{\mathrm{ref}}\left(\mathbf{x}_{i}, \mathbf{t}_{i}+\tau_{0}\right)\right]^{2}
$$

where $\mathbf{x}_{i}$ and $\mathbf{t}_{i}$, as above, correspond to the $i$-th trace location and the respective wavefrontconsistent traveltime and $\delta t$ is a time window centered around the data point in which the coherent waveforms are compared. To accurately preserve conflicting event dips, the misfit function $\Psi\left(\alpha_{0}, \tau_{0}\right)$ compares data and the adapted reflection stack not only on a a single trace, but within an aperture spanning many neighboring trace locations $\mathbf{x}_{i}$. This data aperture, fully analogous to the process of coherent summation, can be pre-defined by the user and its size should commonly exceed the lateral extent of the region, in which the two interfering wavefields intersect.

Figure 3 illustrates for the central trace of the data example shown in Figure 1, that the interfering energy is one order of magnitude weaker than the dominant wavefield and, therefore, can only be preserved by performing the adaptation coherently within an aperture. As expression (11), depending on the data amplitudes, can take a large range of values, a normalized measure of misfit helps to compare adaptation results for different datasets and wavefields. The quantity

$$
\psi\left(\alpha_{0}, \tau_{0}\right)=\frac{\Psi\left(\alpha_{0}, \tau_{0}\right)}{\Psi(1,0 \mathrm{~s})}
$$

i.e. the absolute misfit for the adapted reflection stack divided by the misfit of the unadapted reflection stack only takes values between 0 corresponding to full subtraction and 1 indicating a significant non-vanishing misfit. While in the first scenario the adaptation was likely successful and no interfering events are present, the latter suggests that either the adaptation was entirely unsuccessful, or that within the considered aperture, strong interfering 
events are present which are not honored by the reflection moveout $\Delta t_{\text {ref }}\left(\Delta \mathbf{x}_{i}\right)$. The adaptation process, just as the semblance maximization (3) can be phrased as an optimization problem

$$
\min _{\alpha_{0}, \tau_{0}} \psi\left(\alpha_{0}, \tau_{0}\right)
$$

which is likewise solved for every data point. In contrast to the optimization of the coherence in the original data, however, problem (13) is highly local, since, usually, the reflection stack is already quite accurate and only requires small perturbations of the starting values $\left(\alpha_{0}, \tau_{0}\right)=(1,0 \mathrm{~s})$. Therefore, as is the case in the current implementation, it can be approached with a simplex search (Nelder and Mead, 1965) or equivalent local solvers. Finally, as is also illustrated in Figure 1, an adapted version of equation (10), i.e.

$$
\mathcal{C}_{\text {diff }}\left(\mathbf{x}_{0}, t_{0}\right)+\mathcal{N}\left(\mathbf{x}_{0}, t_{0}\right)=\mathcal{D}\left(\mathbf{x}_{0}, t_{0}\right)-\alpha_{0} \mathcal{C}_{\text {ref }}\left(\mathbf{x}_{0}, t_{0}+\tau_{0}\right)
$$

leads to a good approximation of a diffraction-only dataset. In cases of strong randomnoise contamination, the result of the coherent reflection subtraction might be dominated by the second term on the left-hand side and the usually faint diffracted arrivals might, if at all, barely rise above noise level. While this can be considered a fundamental problem of weak-wavefield prospecting, the suggested first step of semblance maximization and stacking might likewise be applied to the diffraction-only volume to increase the signal-to-noise ratio.

[Figure 4 about here.]

[Figure 5 about here.]

As can be shown for the simple synthetic example introduced in Figure 2, the coherent subtraction (14) depends on a stable and reliable estimation of the aperture consistent 
scaling coefficients $\alpha_{0}$ and time shifts $\tau_{0}$. Generally, as a rule of thumb, the more data is compared, i.e. the larger the data aperture and chosen time windows $\delta t$ are, in which the reflection stack and the input data are compared, the less artifact- and residual-prone the subtraction result becomes. Figure 4 and Figure 5 show the subtraction result, i.e. the separated diffracted wavefield and the corresponding normalized coherent subtraction misfit (12) for different aperture and time window sizes, respectively. As can be observed, small apertures and time windows reveal residual reflected energy and loose detail in the apex region, which can be explained by the fact, that the first-order (slope) subset of equation (6) was used for summation and subtraction. This undesired effect can, in principle, be circumvented by employing a curvature-dependent wavefront filter, as e.g. presented in the form of equations (7b) and (8). However, as the subtraction results for large apertures and time windows likewise indicate, an appropriate choice of these parameters helps to ensure that no valuable portions of the diffracted energy near the apex are lost during summation, even if a rather non-discriminative operator and filter are chosen for the separation near the apex, where reflection and diffraction is tangential. while coherent summation can be viewed as constructive self-interference of a particular event, the coherent subtraction process helps to de-interfere different wavefield components.

\section{EXAMPLES}

In the following, I seek to demonstrate that the proposed fully data-driven wavefield separation method enables to uncover even the faintest diffracted contributions without harming their amplitude and phase content, therefore enabling them to be used in subsequent imaging and inversion workflows. By means of challenging synthetic pre- and poststack examples, as well as a 3D seismic data example, I will illustrate that the presented frame- 
work is reasonably flexible to deal with different types of (dense) data acquisitions and configurations. Through a ground-penetrating radar (GPR) field dataset, I demonstrate that the presented method is not limited to seismic applications but can likewise naturally be applied to electromagnetic waveform data.

\section{Pre- \& poststack seismic data}

[Figure 6 about here.]

[Figure 7 about here.]

[Figure 8 about here.]

To show that the proposed technique works in a controlled but reasonably complex environment, I chose the Sigsbee 2A synthetic dataset, which was developed and made available to the scientific community by the Subsalt Multiples Attenuation and Reduction Technology Joint Venture (SMAART JV). It arose from an industry-scale multi-channel acquisition with source-receiver distances up to almost $8 \mathrm{~km}$ that was simulated in a structurally complex setting mimicking the Sigsbee escarpment below the Gulf of Mexico. The geological dominating feature represents a complex-shaped salt body embedded in a tectonically active sedimentary regime. In addition to a variety of faults, the underlying model is characterized by rugged salt-sediment interfaces with high impedance contrasts. Somewhat unintentionally, owing to the discreteness of the model that was used to simulate the data using constant-density finite-difference modeling, the stair-stepping that can be observed at inclined interfaces generates a very rich but normally hidden diffracted wavefield, which makes this dataset very challenging and suitable to investigate the potential of this method. 
Figure 6(a) shows the result of a common-midpoint stack, which generally preserves interfering wavefields and forms the starting point for the $2 \mathrm{D}$ poststack application. While the mostly horizontal or sub-horizontal features correspond to sedimentary reflections, the steeply dipping complicated and amplitude-strong features in the central part of the dataset largely resulted from diffraction that occurred at the top of the salt body. In Figure 6(b), the coherent subtraction result is displayed. As can be observed, owing to the employment of a curvature-utilizing wavefront filter (equation (8)), the dominant diffracted arrivals connected to the salt body were not harmed in the process, but remain fully preserved. Also, a very rich diffracted wavefield permeating the rest of the section is revealed. Despite the fact that a relatively strong clip was applied to both sections in Figure 6, many diffractions, due to their faintness are hardly visible. For closer inspection, Figure 7 shows closeups whose locations are indicated by the two frames in Figure 6(b) before and after diffraction separation. As can be observed, the coherent wavefield subtraction scheme proved to be very selective and resulted in practically reflection-free data while fully preserving the full amplitude and phase characteristics of the diffracted contributions. To illustrate the workings of the suggested method, Figure 8 compares the maximized semblance norm (a), the corresponding absolute slope measurements (b) and the midpoint-offset curvature ratio expressed by the exponential term in function (8) (Dell and Gajewski, 2011). While the coherence section indicates that in the sedimentary regime, reflection was almost exclusively favored by the semblance maximization, the prominent salt-body diffractions, which reveal high absolute slope values and similar wavefront curvature estimates in the CMP and the zero-offset domain, are well-distinguishable from the reflected contributions. Accordingly, they could be preserved in the coherent subtraction.

[Figure 9 about here.] 


\section{$3 \mathrm{D}$ seismic data}

[Figure 10 about here.]

Here, I seek to demonstrate that the process of coherent wavefield subtraction can likewise be applied to 3D seismic data. Similar to the Sigsbee 2A synthetic dataset which was chosen for the controlled pre- and poststack seismic examples in the previous subsection, the SEG/EAGE salt model narrow-azimuth dataset was deemed well-suited to simulate a challenging multi-channel data acquisition with amplitude-strong signatures due to salt coexisting with smooth reflections connected to sub-horizontal sedimentary layering (Aminzadeh et al., 1995). It was generated in a collaborative effort by the Advanced Computational Technology Initiative, with the model being constructed by the SEG research committee 
and modeling performed by the Sandia and Los Alamos National Laboratories. Similar to Sigsbee $2 \mathrm{~A}$, the simulated scenario incorporates faults that are connected to salt-related tectonics and result in faint diffractions which are accompanied by rich but even weaker back-scattering due to model coarseness.

In order to complement the previous figures and to emphasize the $3 \mathrm{D}$ character of diffraction, Figure 10 compares a common-midpoint stacked volume, which served as the starting point, the approximated reflected wavefield, the coherent subtraction result, and the corresponding normalized misfit (12) for the time slice $1.8 \mathrm{~s}$. For the sake of simplicity, the first-order subset of the 3D zero-offset approximation (6) and the slope-based wavefront filter (equation (7a)) were used to perform the coherent wavefield subtraction. However, as was demonstrated in Figure 4, a sufficiently large data aperture and time window can help overcome the conventional limitations and allow to fully preserve diffracted features with high curvature. Small data gaps in Figure 10(b) indicate where primary diffracted energy was detected by the slope-based wavefront filter (7a). Low apparent wavelengths are normally connected to reflection in time slices and, consequently, almost entirely vanish after the subtraction. Generally the detected diffracted wavefield has a high degree of complexity. However, closer inspection of Figure 10(c) reveals circular features related to localized diffraction occurring at the flank of the predominant salt body. The bright colors in the subtraction misfit suggest that the process of semblance maximization and coherent summation leading to 10 (b) suppresses a detail-rich background wavefield - dominated by diffraction - which is commonly ignored in seismic processing. 


\section{Ground-penetrating radar data}

[Figure 11 about here.]

The presented scheme builds on the concept of coherence, a property that, from a theoretical viewpoint, can be attributed to arbitrary wavefields. In fact, its rigorous treatment was first carried out for light, i.e. the visible spectrum of electromagnetic waves (Born and Wolf, 2013). Accordingly, it is not surprising that the presented method for wavefield separation can be tailored and applied to a wide range of geophysical wave phenomena, including ground-penetrating (GPR) radar measurements. In contrast to seismology, GPR wavefields are naturally limited in how deep they can penetrate the Earth, predominantly due to the fact that strong attenuation occurs in the upper portions of the crust. In addition, due to practical limitations, their controlled sources usually operate at higher frequencies and, consequently lower wavelengths, ultimately resulting in higher resolution (e.g. Telford et al., 1990). Since the near surface is structurally complex, GPR antennas often record complicated and highly interfering back-scattered wavefields. Additionally, GPR data are cost-effective to acquire resulting in dense wavefield sampling - as mentioned earlier, the main prerequisite for the proposed scheme to be applicable.

In line with the presented seismic data examples, I chose a complex field dataset consisting of several lines that were recorded on Long Beach Island by the US Geological Survey (Zaremba et al., 2016). Just like in the seismic poststack case, these GPR data were acquired in the traditional zero-offset configuration, i.e. with coinciding emitting and receiving antenna positions. However, as the presented framework was demonstrated to be also applicable to multi-channel acquisitions, modern finite-offset GPR measurements are likewise expected to benefit from the presented wavefield separation framework. Figure 11 
compares the input data, the diffraction separation result and the subtraction misfit for one of the available GPR lines. While in particular very close to the acquisition surface, prominent diffraction patterns originate, non-Snell back-scattering can likewise be observed for later arrival times. The misfit section reveals where diffracted signatures are hiding behind the more dominant reflections. Generally, across the whole recorded line, faint but highly illuminating wavefields could be uncovered, which are now accessible and exploitable for further investigations.

\section{DISCUSSION}

[Figure 12 about here.]

[Figure 13 about here.]

The presented scheme for diffraction separation is quite universal and should lead to interesting new applications in different fields. Although I tried to develop the underlying theory as rigorously and general as possible, a variety of implementational aspects can surely be improved. From a practical viewpoint, just as in conventional coherent stacking, the choice of the right data aperture to perform the attribute estimation allowing the formulation of supportive wavefront filters, and, in particular, during the coherent subtraction process, is not always trivial and depends on a variety of factors, including data sparsity, channel-count, frequency content, wavefield complexity, and the moveout operators employed. While a sufficiently large data aperture was demonstrated to increase the selectivity of the separation, it can be argued that a too large aperture in conjunction with a relatively simple operator can lead to degraded subtraction results and residual reflected energy (as can, upon closer inspection, e.g. be observed in some parts of Figure 6(b)). Al- 
though in the presented examples the separation turned out to be robust in most scenarios, a successful extraction of diffraction-only data can not be guaranteed. However, there also exist ways to overcome or weaken these limitations. On the one hand, other more accurate moveout approximations, e.g. explicitly addressing overburden anisotropy, can be employed (e.g. Grechka and Tsvankin, 1998; Fomel and Stovas, 2010; Sripanich et al., 2017), on the other, the coherent wavefield subtraction can be applied in an iterative fashion. Since, however, reflected rather than diffracted events are targeted, a generalized hyperbolic expression was deemed sufficient for most cases.

Although not specifically addressed here, the combination of full-prestack data enhancement and regularization with zero-offset attribute estimation and processing for diffraction imaging, as suggested by Bakhtiari Rad et al. (2018) provides a highly efficient and stable alternative to the presented generic full-prestack coherence analysis. Since, in contrast to Bakhtiari Rad et al. (2018), the reflected rather than the diffracted wavefield is targeted, the use of hyperbolic 3D moveout operators such as (6) seems more reasonable and less prone to errors and inaccuracies. In general, earlier ideas for wavefront-based diffraction separation and imaging, such as e.g. developed by Dell and Gajewski (2011) and Bakhtiari Rad et al. (2018) can, in principle, be fully incorporated in the presented coherent subtraction framework. For example, the formulation of additional, more selective and better-informed wavefront filters, as e.g. suggested by Schwarz and Gajewski (2017a) forms a very potent direction of future research, as the preservation of amplitude-strong diffraction crucially depends on them. First synthetic results have indicated, that the employment of a median filter in the formulation of the misfit function (11) can lead to an improved separation result in certain configurations. Taking inspiration from techniques used in image segmentation, Bauer et al. (2018) discuss automated strategies to discriminate and isolate individual 
diffractions for wavefront tomographic inversion. The employed discrimination criteria can likewise be naturally incorporated in the presented coherent wavefield subtraction scheme. For the 3D phenomenon of edge diffraction the wavefield can be separated in a reflected (strike-parallel) and point-diffracted (strike-normal) contribution. As the workflow builds on the illuminating and weak character of point diffraction, only the latter portion is reliably retrievable using the introduced coherence and wavefront arguments. However, as it is precisely this contribution that bears the potential for improved resolution and illumination in imaging and inversion, this should not be viewed as a principal restriction.

Generally, I argue that the suggested coherent subtraction strategy represents a largely automatable framework for diffraction separation in arbitrary media that bears the potential for targeted diffraction imaging and inversion. For illustration, Figure 12 and Figure 13 show a diffraction image resulting from the separated wavefield of the Sigsbee 2A synthetic dataset. As can be seen in Figure 13(b), conventional Kirchhoff-type (time) migration of the unseparated data accurately images laterally continuous features, while the diffraction image largely resolves every stair step in the discrete velocity model shown in Figure 13(a), which was used for the finite-difference modeling. In contrast to migration-related techniques, the separation is performed directly in the data domain, thereby preserving the diffraction's moveout, phase and amplitude characteristics, which makes it attractive for diffractionbased velocity model building (e.g. Fomel et al., 2007; Reshef and Landa, 2009; Santos et al., 2012; Bauer et al., 2017). The introduced framework is reasonably flexible, as new filter functions can be formulated that specifically target other forms of undesired interfering coherent wavefield components, such as e.g. surface-related multiple reflections, surface waves, ground roll, or even amplitude-strong transmitted wavefields to uncover faint backscattering in earthquake studies. Likewise, the application of the presented method to the 
de-blending of data arising from simultaneous-source acquisitions should be considered and investigated.

\section{CONCLUSIONS}

I have presented a detailed account of a new largely automatable process for pre- and poststack $2 \mathrm{D}$ and $3 \mathrm{D}$ seismic and ground-penetrating radar (GPR) diffraction separation. The method builds on the assumption that data were acquired densely and that even complicated interfering wavefields can be viewed as a superposition of locally coherent contributions. While previous, coherence-based studies were directly focusing on accurately approximating diffraction moveout and coherently summing along these operators, the suggested strategy aims at sufficiently describing the other more dominant wavefield components, in particular reflections, and subtract them from the input data. To preserve hidden diffracted energy when conflicting event dips are recorded, this subtraction process is performed in an adaptive and aperture-consistent fashion. Forming a central ingredient of the proposed scheme, the semblance norm is maximized in a vicinity around each investigated data point. As a by-product, wavefront attributes are estimated, which can inform the subsequent coherent subtraction process through data-derived wavefront filters. Realistic synthetic $2 \mathrm{D}$ and $3 \mathrm{D}$ multi-channel as well as GPR field data examples revealed that the suggested framework for wavefield separation is readily applicable to a wide range of data configurations and bears the potential to successfully extract even extremely amplitude-weak diffractions that were not accessible using conventional summation methods and make them usable for dedicated processing. 


\section{ACKNOWLEDGMENTS}

I thankfully acknowledge financial support through a research fellowship of the German Research Foundation (DFG, SCHW 1870/1-1). In addition, I thank Alexander Bauer, Dirk Gajewski and Leon Diekmann for ongoing and fruitful discussions on the topic. I am grateful to Paula Koelemeijer and Jeffrey Winterbourne, Alistair Crosby, and Joseph Dellinger at BP for encouraging feedback before and during my visit in Sunbury. Karin Sigloch and Tarje Nissen-Meyer provided important support to further pursue this direction of research. John Etgen, Dmitrii Merzlikin, an anonymous reviewer and the responsible associate editor greatly helped to improve the paper. Concluding thanks go to the Subsalt Multiples Attenuation and Reduction Technology Joint Venture (SMAART JV), Sandia and Los Alamos National Laboratories and SEG for generating the synthetic data, as well as the US Geological Survey (USGS) for making the GPR field data from New Jersey publicly available. 


\section{REFERENCES}

Ahmed, K., B. Schwarz, and D. Gajewski, 2015, Application of the 3D common-reflectionsurface stack workflow in a crystalline rock environment: Geophysical Prospecting, 63, 990-998.

Aminzadeh, F., N. Burkhard, T. Kunz, L. Nicoletis, and F. Rocca, 1995, 3-D modeling project: 3rd report: The Leading Edge, 14, 125-128.

Asgedom, E. G., L. J. Gelius, and M. Tygel, 2012, Seismic coherency measures in case of interfering events: A focus on the most promising candidates of higher-resolution algorithms: IEEE Signal Processing Magazine, 29, 47-56.

Bakhtiari Rad, P., B. Schwarz, D. Gajewski, and C. Vanelle, 2018, Common-reflectionsurface-based prestack diffraction separation and imaging: Geophysics, 83, S47-S55.

Barros, T., R. Ferrari, R. Krummenauer, and R. Lopes, 2015, Differential evolution-based optimization procedure for automatic estimation of the common-reflection surface traveltime parameters: Geophysics, 80, WD189-WD200.

Bauer, A., B. Schwarz, and D. Gajewski, 2016, Enhancement of prestack diffraction data and attributes using a traveltime decomposition approach: Studia Geophysica et Geodaetica, 60, $471-486$.

— 2017 , Utilizing diffractions in wavefront tomography: Geophysics, 82, R65-R73.

Bauer, A., B. Schwarz, T. Werner, and D. Gajewski, 2018, Unsupervised global identification of diffractions and passive events using data-derived wavefront attributes: Geophysical Journal International, submitted.

Baykulov, M., and D. Gajewski, 2009, Prestack seismic data enhancement with partial common-reflection-surface (CRS) stack: Geophysics, 74, V49-V58.

Bergler, S., P. Hubral, P. Marchetti, A. Cristini, and G. Cardone, 2002, 3D common- 
reflection-surface stack and kinematic wavefield attributes: The Leading Edge, 21, 10101015.

Berkovitch, A., I. Belfer, Y. Hassin, and E. Landa, 2009, Diffraction imaging by multifocusing: Geophysics, 74, WCA75-WCA81.

Born, M., and E. Wolf, 2013, Principles of optics: electromagnetic theory of propagation, interference and diffraction of light: Elsevier.

Dafni, R., and W. W. Symes, 2017, Diffraction imaging by prestack reverse-time migration in the dip-angle domain: Geophysical Prospecting, 65, 295-316.

Dell, S., and D. Gajewski, 2011, Common-reflection-surface-based workflow for diffraction imaging: Geophysics, 76, S187-S195.

Fomel, S., 2002, Applications of plane-wave destruction filters: Geophysics, 67, 1946-1960.

—, 2009, Velocity analysis using AB semblance: Geophysical Prospecting, 57, 311-321.

Fomel, S., E. Landa, and M. T. Taner, 2007, Poststack velocity analysis by separation and imaging of seismic diffractions: Geophysics, 72, U89-U94.

Fomel, S., and A. Stovas, 2010, Generalized nonhyperbolic moveout approximation: Geophysics, 75, U9-U18.

Gelchinsky, B., A. Berkovitch, and S. Keydar, 1999a, Multifocusing homeomorphic imaging: Part 1. Basic concepts and formulas: Journal of applied geophysics, 42, 229-242.

— 1999b, Multifocusing homeomorphic imaging: Part 2. Multifold data set and multifocusing: Journal of Applied Geophysics, 42, 243-260.

Grechka, V., and I. Tsvankin, 1998, 3-D description of normal moveout in anisotropic inhomogeneous media: Geophysics, 63, 1079-1092.

Guitton, A., and D. Verschuur, 2004, Adaptive subtraction of multiples using the 11-norm: Geophysical Prospecting, 52, 27-38. 
Höcht, G., P. Ricarte, S. Bergler, and E. Landa, 2009, Operator-oriented CRS interpolation: Geophysical Prospecting, 57, 957-979.

Hubral, P., 1983, Computing true amplitude reflections in a laterally inhomogeneous earth: Geophysics, 48, 1051-1062.

Jäger, R., J. Mann, G. Höcht, and P. Hubral, 2001, Common-reflection-surface stack: Image and attributes: Geophysics, 66, 97-109.

Khaidukov, V., E. Landa, and T. J. Moser, 2004, Diffraction imaging by focusing-defocusing: An outlook on seismic superresolution: Geophysics, 69, 1478-1490.

Klokov, A., and S. Fomel, 2012, Separation and imaging of seismic diffractions using migrated dip-angle gathers: Geophysics, 77, S131-S143.

Kozlov, E., N. Barasky, E. Korolev, A. Antonenko, and E. Koshchuk, 2004, Imaging scattering objects masked by specular reflections, in SEG Technical Program Expanded Abstracts 2004: Society of Exploration Geophysicists, 1131-1134.

Krey, T., 1952, The significance of diffraction in the investigation of faults: Geophysics, 17, $843-858$.

Landa, E., and S. Keydar, 1998, Seismic monitoring of diffraction images for detection of local heterogeneities: Geophysics, 63, 1093-1100.

Mann, J., 2001, Common-reflection-surface stack and conflicting dips, in SEG Technical Program Expanded Abstracts 2001: Society of Exploration Geophysicists, 1886-1889.

Mayne, W. H., 1962, Common reflection point horizontal data stacking techniques: Geophysics, 27, 927-938.

Merzlikin, D., T. A. Meckel, S. Fomel, and Y. Sripanich, 2017, Diffraction imaging of high-resolution 3D P-cable data from the Gulf of Mexico using azimuthal plane-wave destruction: First Break, 35, 35-41. 
Moser, T., and C. Howard, 2008, Diffraction imaging in depth: Geophysical Prospecting, 56, $627-641$.

Müller, N.-A., 2009, Treatment of conflicting dips in the 3D common-reflection-surface stack: Geophysical Prospecting, 57, 981-995.

Neidell, N. S., and M. T. Taner, 1971, Semblance and other coherency measures for multichannel data: Geophysics, 36, 482-497.

Nelder, J. A., and R. Mead, 1965, A simplex method for function minimization: The Computer Journal, 7, 308-313.

Reshef, M., and E. Landa, 2009, Post-stack velocity analysis in the dip-angle domain using diffractions: Geophysical Prospecting, 57, 811-821.

Santos, L. A., W. J. Mansur, and G. A. McMechan, 2012, Tomography of diffraction-based focusing operators: Geophysics, 77, R217-R225.

Schleicher, J., M. Tygel, and P. Hubral, 1993, Parabolic and hyperbolic paraxial two-point traveltimes in 3D media: Geophysical Prospecting, 41, 495-513.

Schwarz, B., and D. Gajewski, 2017a, A generalized view on normal moveout: Geophysics, 82, V335-V349.

$\longrightarrow, 2017 \mathrm{~b}$, Accessing the diffracted wavefield by coherent subtraction: Geophysical Journal International, 211, 45-49.

Soleimani, M., I. Piruz, J. Mann, and P. Hubral, 2009, Common-Reflection-Surface stack: accounting for conflicting dip situations by considering all possible dips: Journal of Seismic Exploration, 18, 271.

Sripanich, Y., S. Fomel, A. Stovas, and Q. Hao, 2017, 3D generalized nonhyperboloidal moveout approximation: Geophysics, 82, C49-C59.

Storn, R., and K. Price, 1997, Differential evolution-a simple and efficient heuristic for global 
optimization over continuous spaces: Journal of Global Optimization, 11, 341-359.

Taner, M. T., and F. Koehler, 1969, Velocity spectraDigital computer derivation applications of velocity functions: Geophysics, 34, 859-881.

Telford, W. M., W. Telford, L. Geldart, R. Sheriff, and R. E. Sheriff, 1990, Applied Geophysics: 1.

Ursin, B., 1982, Quadratic wavefront and traveltime approximations in inhomogeneous layered media with curved interfaces: Geophysics, 47, 1012-1021.

Verschuur, D. J., A. Berkhout, and C. Wapenaar, 1992, Adaptive surface-related multiple elimination: Geophysics, 57, 1166-1177.

Walda, J., and D. Gajewski, 2017, Determination of wavefront attributes by differential evolution in the presence of conflicting dips: Geophysics, 82, V229-V239.

Xie, Y., and D. Gajewski, 2017, 5-D interpolation with wave-front attributes: Geophysical Journal International, 211, 919-941.

Yin, J., and N. Nakata, 2017, Diffraction Imaging with Geometric-mean Reverse Time Migration, in SEG Technical Program Expanded Abstracts 2017: Society of Exploration Geophysicists, 974-979.

Zaremba, N. J., K. E. Smith, J. M. Bishop, and C. G. Smith, 2016, Ground-penetrating radar and differential global positioning system data collected from Long Beach Island, New Jersey, April 2015: Technical report, US Geological Survey.

Zhang, Y., S. Bergler, and P. Hubral, 2001, Common-reflection-surface (CRS) stack for common offset: Geophysical Prospecting, 49, 709-718. 


\section{LIST OF FIGURES}

1 Illustration of the coherent subtraction scheme. Amplitude-strong coherent wavefields are usually favored, when interfering with weak events. Here, I suggest to approximate the dominating contribution through coherent summation before adaptively subtracting it from the input data. . . . . . . . . .

2 A small (clipped) synthetic data example where edge diffraction, due to stairstepping at the interfaces, heavily interferes with coherent reflection events (a). Automated coherence analysis naturally favors the amplitude-strong reflections, whereas the faint diffraction background is lost in the process (b). To allow for coherent subtraction, small local amplitude scaling coefficients $\alpha_{0}$ (c) and time shifts $\tau_{0}(\mathrm{~d})$ are estimated within a data aperture centered at each data point $\left(\mathbf{x}_{0}, t_{0}\right) \ldots \ldots \ldots \ldots$

3 Closeup of the central trace of the illustrative data example shown in Figure 1. Shown are the actual data trace (bold), the coherent stack (dotted) and the adaptive subtraction result representing the weak background wavefield (dashed). . . . . . . . . . . . . . . . . . .

4 Illustration of the influence of (a) the aperture size and (b) the time window length on the diffraction separation for the same simple synthetic example as shown in Figure 2. In both cases, the quality of the subtraction improves when more data points are considered in the coherent adaptation step (compare Figure 1) . . . . . . . . . . . . . . . . . . .

5 The normalized subtraction misfit corresponding to the different configurations considered in Figure 4. For large data windows (aperture or time), characteristic smoothing can be observed, while small apertures and small time windows reveal largely equivalent behavior. . . . . . . . . . . . .

6 (a) Automatically generated (clipped) CMP stack of the Sigsbee 2 A synthetic dataset, simulating the seismic data acquisition in a complex salt-body environment. (b) Diffracted wavefield extracted using the suggested coherent wavefield subtraction scheme. The frames indicate closeup locations which are investigated in more detail. . . . . . . . . . . . . . .

7 Closeups indicated by the frames in Figure 6(b) shown before (left) and after (right) the automated subtraction of the reflected wavefield. Owing to model-coarseness and related stair-stepping at the interfaces, diffraction largely occurred at edges, resulting in an amplitude reversal at the apex - an effect fully preserved in the suggested subtraction scheme. . . . . . . . . .

8 By-products of conventional coherence analysis, as performed e.g. in the frame of the common-reflection-surface (CRS) or the multifocusing stack for the Sigsbee 2A example. Shown are (a) the maximized local wavefield coherence expressed through the semblance norm, (b) the estimated slope field as well as (c) the wavefront curvature ratio - attributes that can be used to identify and preserve diffracted contributions during subtraction. . . . . . . 
9 An excerpt of the Sigsbee 2A prestack data volume illustrating the applicability of the coherent subtraction strategy for more general data configurations. Shown are (a) 100 adjacent raw shot gathers, (b) the maximized semblance norm, (c) the corresponding coherent summation result, and (d) the adaptive subtraction of $(\mathrm{c})$ from $(\mathrm{a}) . \ldots \ldots \ldots \ldots$

10 Illustration of the applicability of the coherent wavefield subtraction strategy for the 3D SEG/EAGE salt model seismic dataset. Shown are the $1.8 \mathrm{~s}$ time slices of (a) the input data, (b) the adapted reflection stack, (c) the coherent subtraction result, and (d) the normalized coherent subtraction misfit. Gaps in (b) indicate where dominant diffracted energy is detected and preserved through wavefront filters in the subtraction procedure. . . . . . . . . . .

11 Line of a ground-penetrating radar (GPR) field dataset recorded by the US Geological Survey (USGS) on Long Beach Island in 2015 (Zaremba et al., 2016). Displayed are (a) the raw input data, (b) the separation result, and (c) the corresponding estimated misfit between subtracted data and input. Bright colors in (c) indicate where interference between reflection and diffraction occurs. . . . . . . . . . . . . . . . . . . .

12 Diffraction (time) image of the Sigsbee 2A model gained through Kirchhofftype focusing of the coherent wavefield subtraction result shown in Figure 6(b). In addition to dominant diffraction occuring at the top-of-salt, smallscale model discontinuities arising from the discretization of the model are detected. . . . . . . . . . . . . . . . . . . . .

13 Closeup of the diffraction image (c) indicated by the frame in Figure 12, compared to the actual discrete depth model (a) and the conventional image resulting from the migration of the unseparated wavefield (b), in which reflection clearly dominates. The paired uppermost foci in (c) indicate that even the very thin shallow layer at approximately $2 \mathrm{~km}$ depth can be resolved. 44 


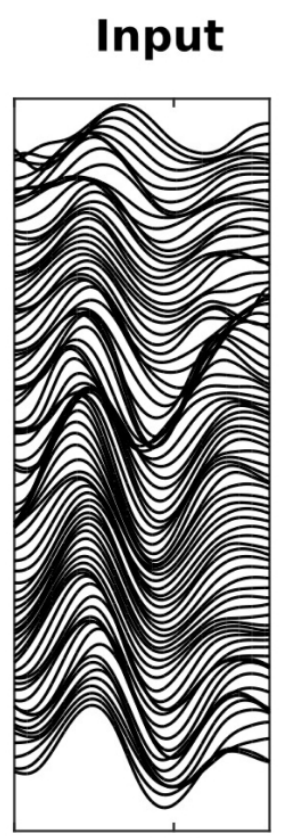

4.1

Time (s)

\section{Coherent adaptation}

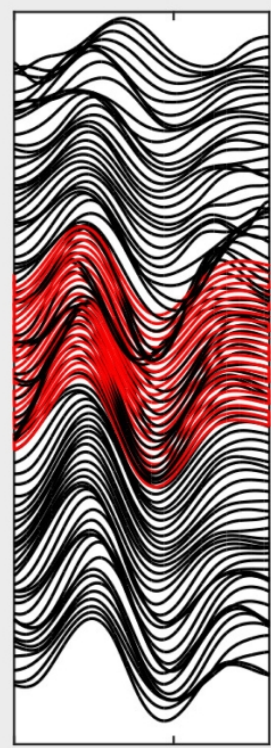

4.1

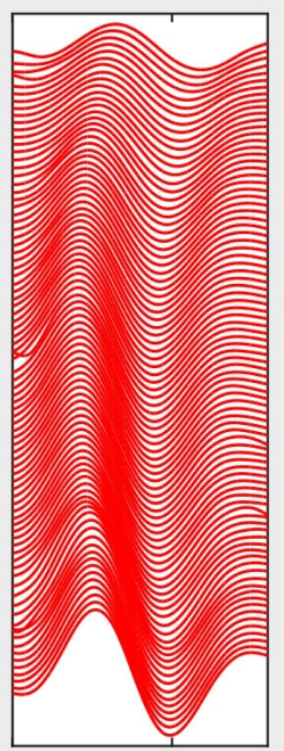

4.1

Time (s)

\section{Subtraction}

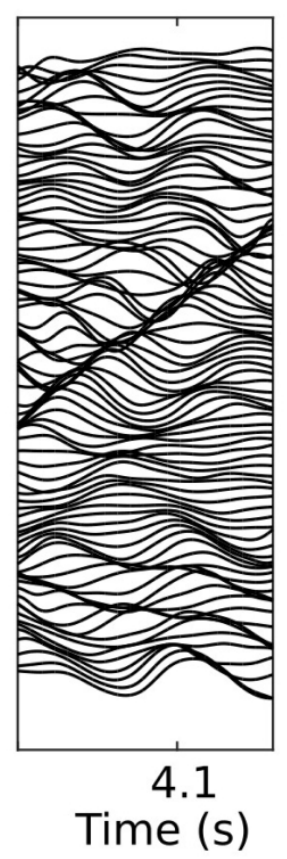

Figure 1. Illustration of the coherent subtraction scheme. Amplitude-strong coherent wavefields are usually favored, when interfering with weak events. Here, I suggest to approximate the dominating contribution through coherent summation before adaptively subtracting it from the input data.

$469 \times 352 \mathrm{~mm}(300 \times 300 \mathrm{DPI})$ 
Figure 2. A small (clipped) synthetic data example where edge diffraction, due to stair-stepping at the interfaces, heavily interferes with coherent reflection events (a). Automated coherence analysis naturally favors the amplitude-strong reflections, whereas the faint diffraction background is lost in the process (b). To allow for coherent subtraction, small local amplitude scaling coefficients $a_{0}(c)$ and time shifts $T_{0}(d)$ are estimated within a data aperture centered at each data point $\left(\mathrm{x}_{0}, \mathrm{t}_{0}\right)$.

$$
610 \times 356 \mathrm{~mm}(300 \times 300 \mathrm{DPI})
$$




\begin{abstract}
Figure 3. Closeup of the central trace of the illustrative data example shown in Figure 1. Shown are the actual data trace (bold), the coherent stack (dotted) and the adaptive subtraction result representing the weak background wavefield (dashed).
\end{abstract}

$84 \times 58 \mathrm{~mm}(300 \times 300$ DPI $)$

\section{(C) 2019 Society of Exploration Geophysicists.}



diffraction separation for the same simple synthetic example as shown in Figure 2 . In both cases, the quality of the subtraction improves when more data points are considered in the coherent adaptation step (compare Figure 1).

$610 \times 367 \mathrm{~mm}(600 \times 600 \mathrm{DPI})$
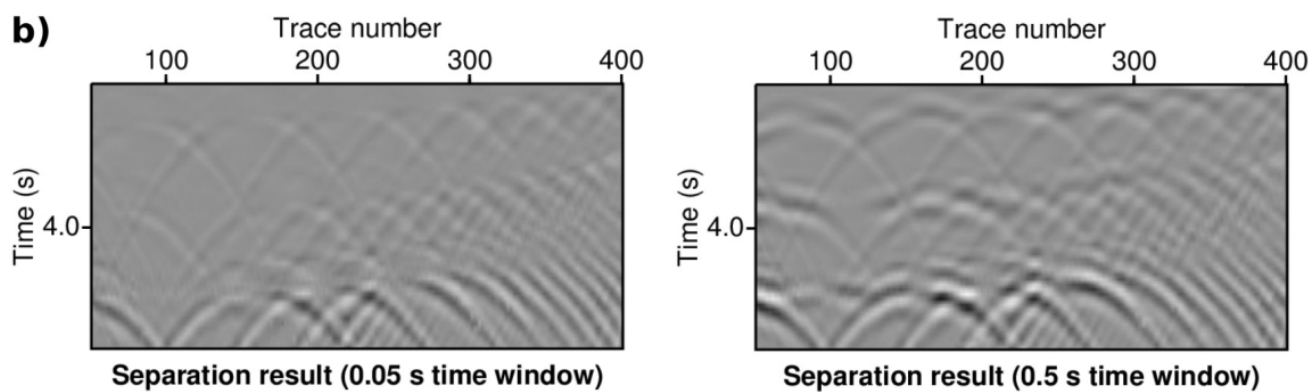

a)
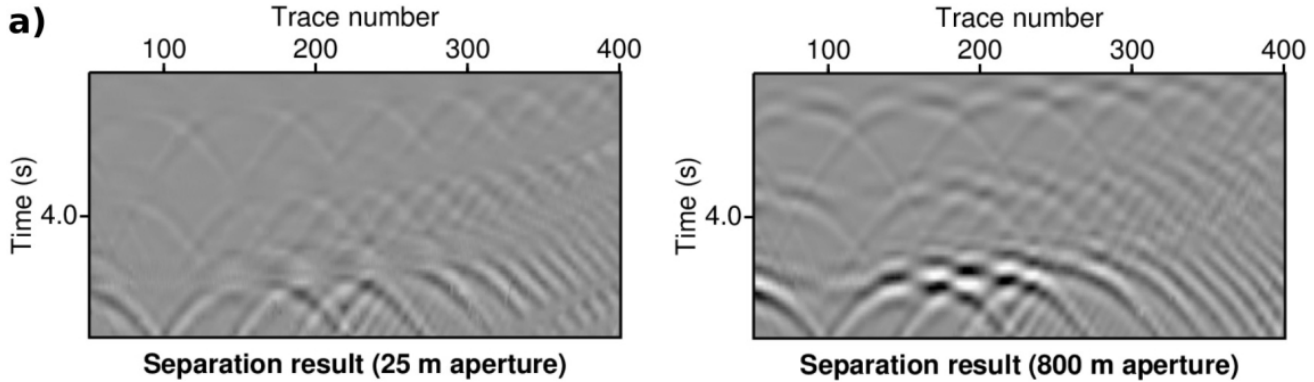

Separation result ( $800 \mathrm{~m}$ aperture)

Separation result $(0.5 \mathrm{~s}$ time window) 

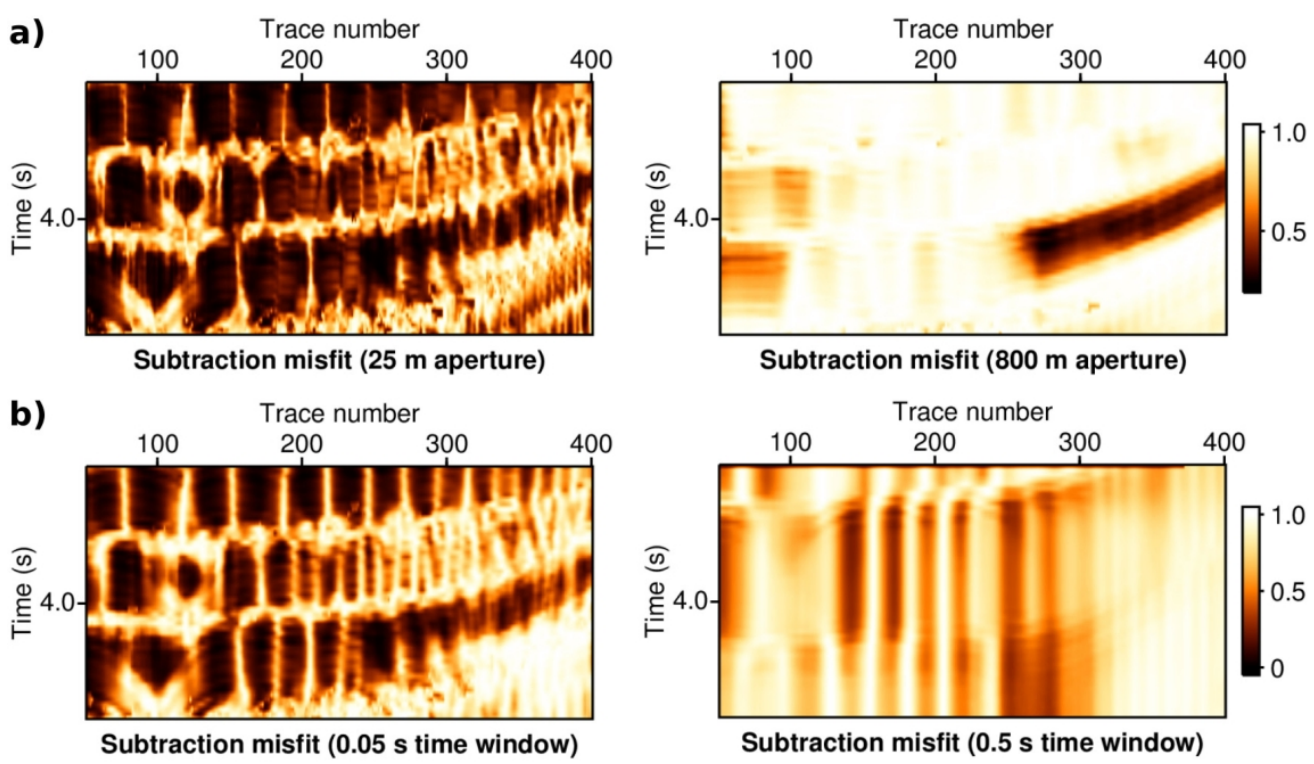

Figure 5. The normalized subtraction misfit corresponding to the different configurations considered in Figure 4. For large data windows (aperture or time), characteristic smoothing can be observed, while small apertures and small time windows reveal largely equivalent behavior.

$610 \times 351 \mathrm{~mm}(300 \times 300 \mathrm{DPI})$ 

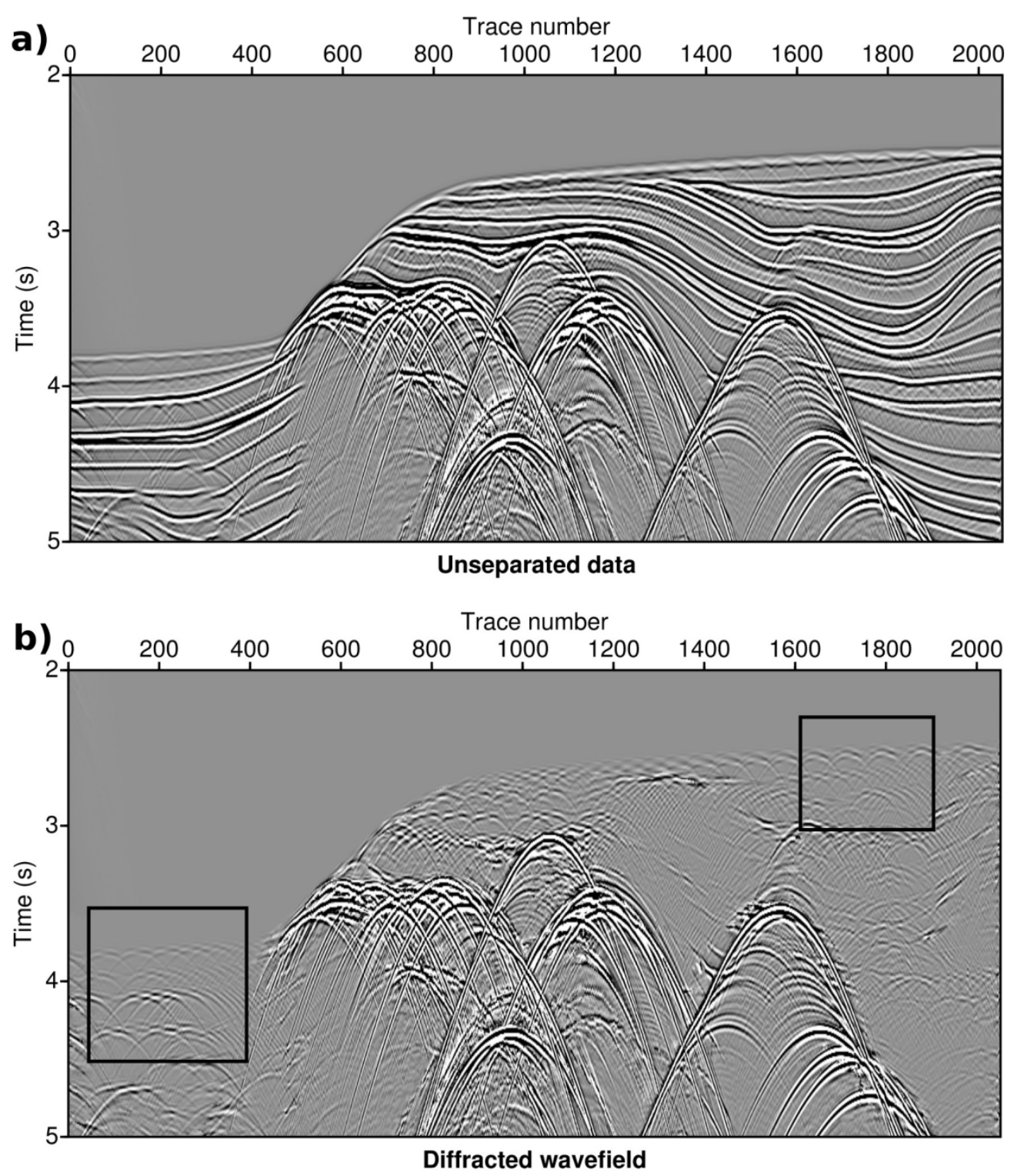

Figure 6. (a) Automatically generated (clipped) CMP stack of the Sigsbee 2A synthetic dataset, simulating the seismic data acquisition in a complex salt-body environment. (b) Diffracted wavefield extracted using the suggested coherent wavefield subtraction scheme. The frames indicate closeup locations which are investigated in more detail.

$610 \times 708 \mathrm{~mm}(600 \times 600 \mathrm{DPI})$ 

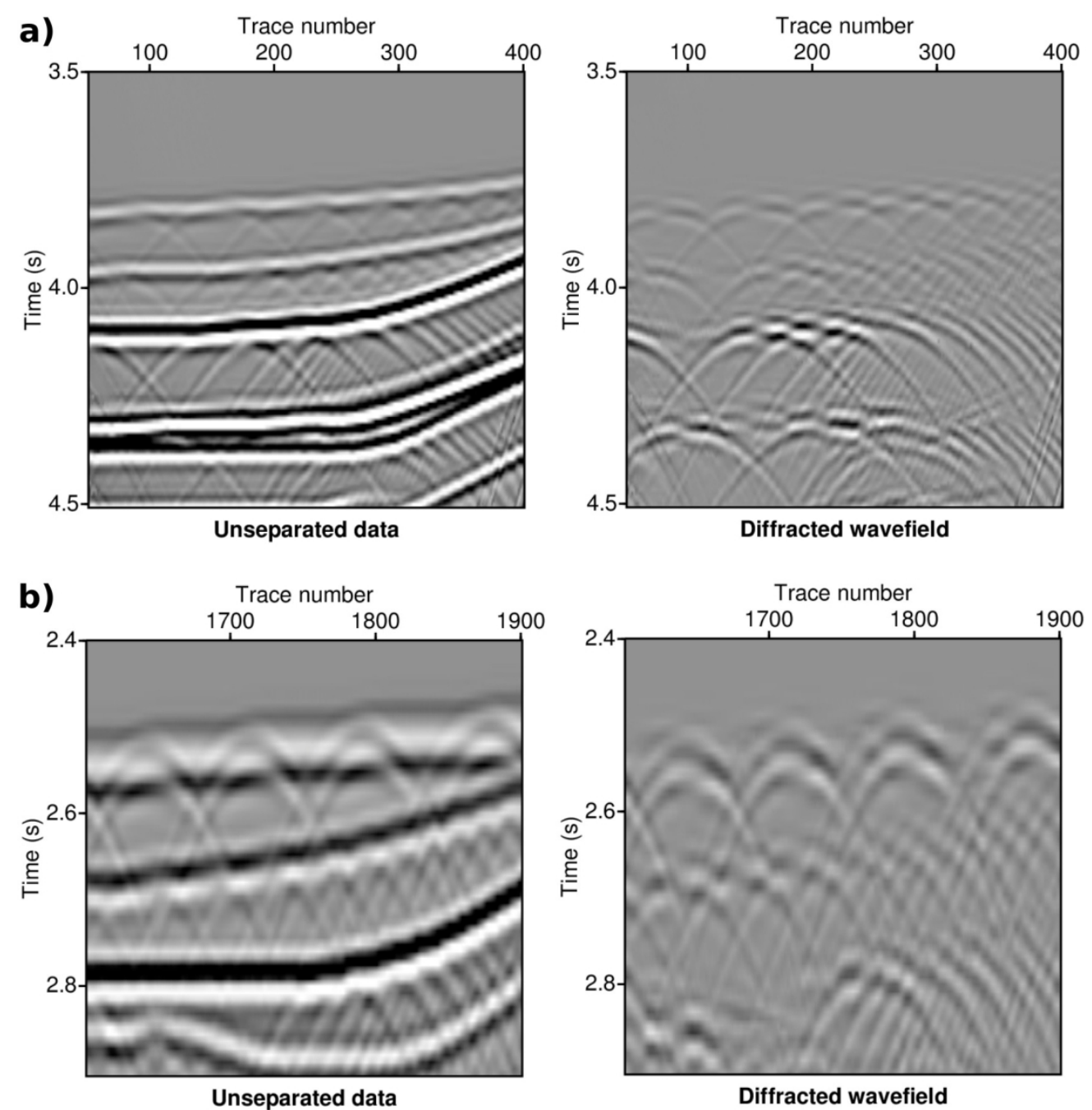

Figure 7. Closeups indicated by the frames in Figure 6(b) shown before (left) and after (right) the automated subtraction of the reflected wavefield. Owing to model-coarseness and related stair-stepping at the interfaces, diffraction largely occurred at edges, resulting in an amplitude reversal at the apex - an effect fully preserved in the suggested subtraction scheme.

\section{$610 \times 630 \mathrm{~mm}(600 \times 600 \mathrm{DPI})$}



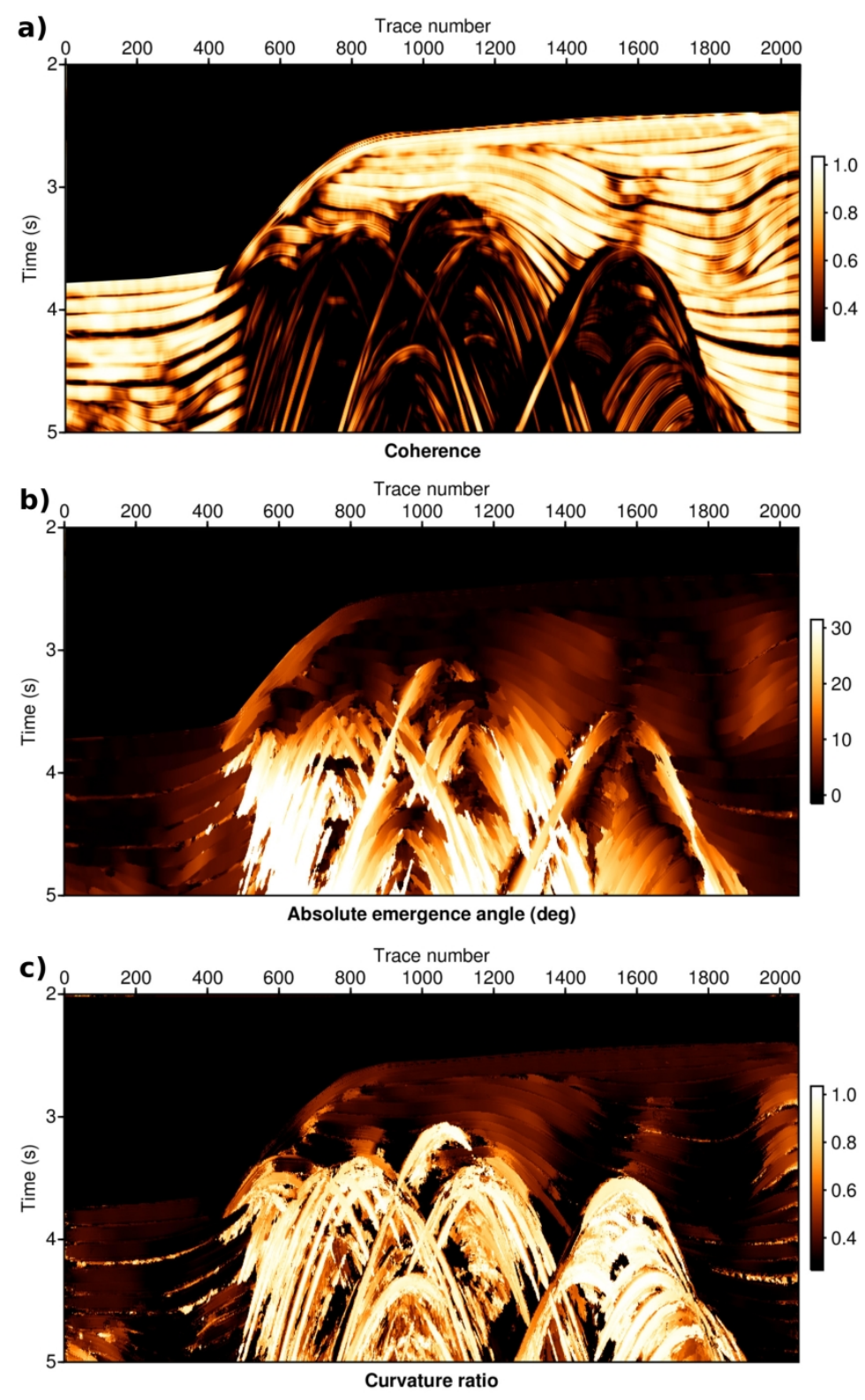

Figure 8. By-products of conventional coherence analysis, as performed e.g. in the frame of the commonreflection-surface (CRS) or the multifocusing stack for the Sigsbee 2A example. Shown are (a) the maximized local wavefield coherence expressed through the semblance norm, (b) the estimated slope field as well as (c) the wavefront curvature ratio - attributes that can be used to identify and preserve diffracted contributions during subtraction.

$610 \times 991 \mathrm{~mm}(600 \times 600 \mathrm{DPI})$ 

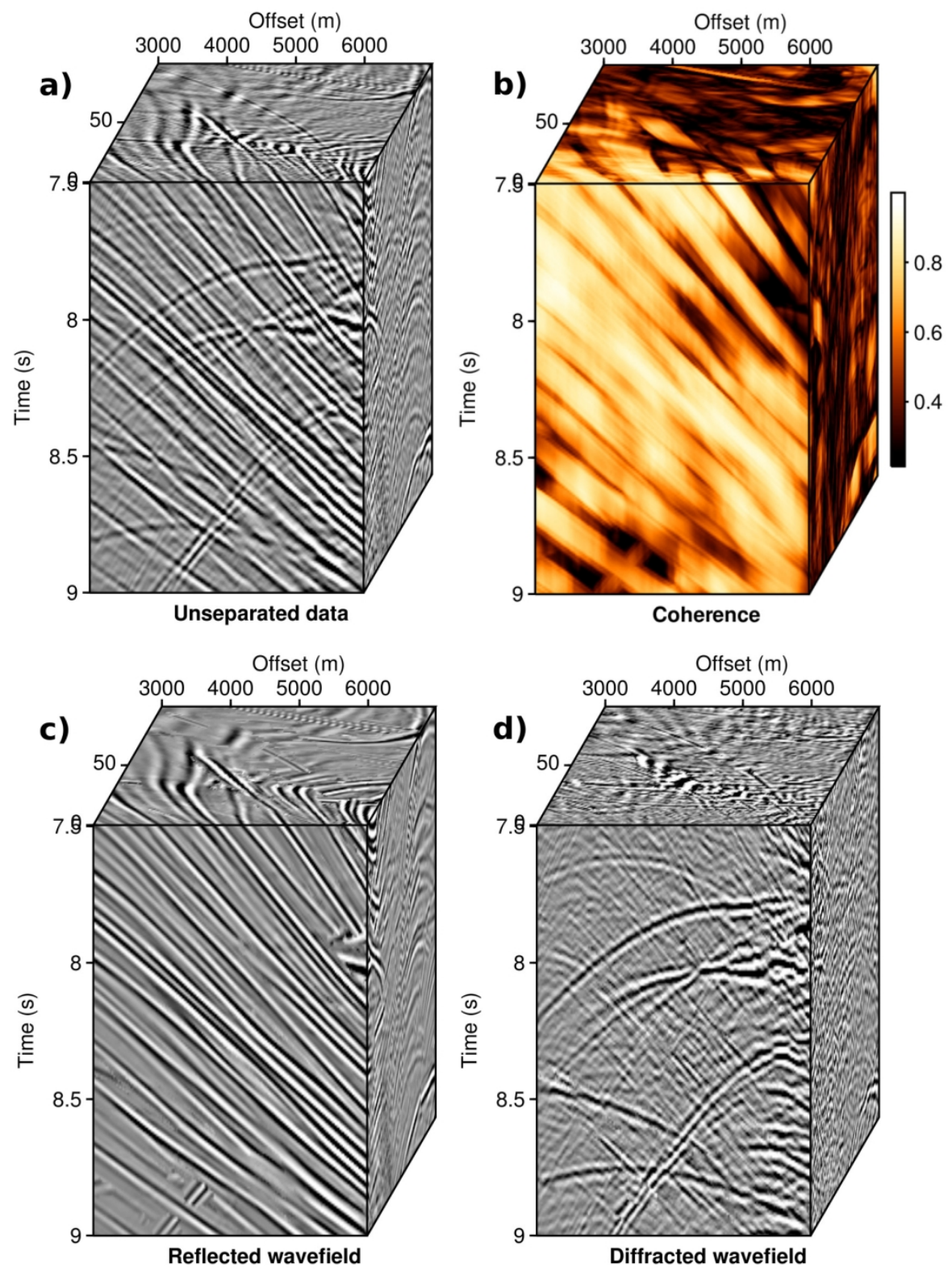

Figure 9. An excerpt of the Sigsbee 2A prestack data volume illustrating the applicability of the coherent subtraction strategy for more general data configurations. Shown are (a) 100 adjacent raw shot gathers, (b) the maximized semblance norm, (c) the corresponding coherent summation result, and (d) the adaptive subtraction of (c) from (a).

$610 \times 821 \mathrm{~mm}(600 \times 600 \mathrm{DPI})$ 

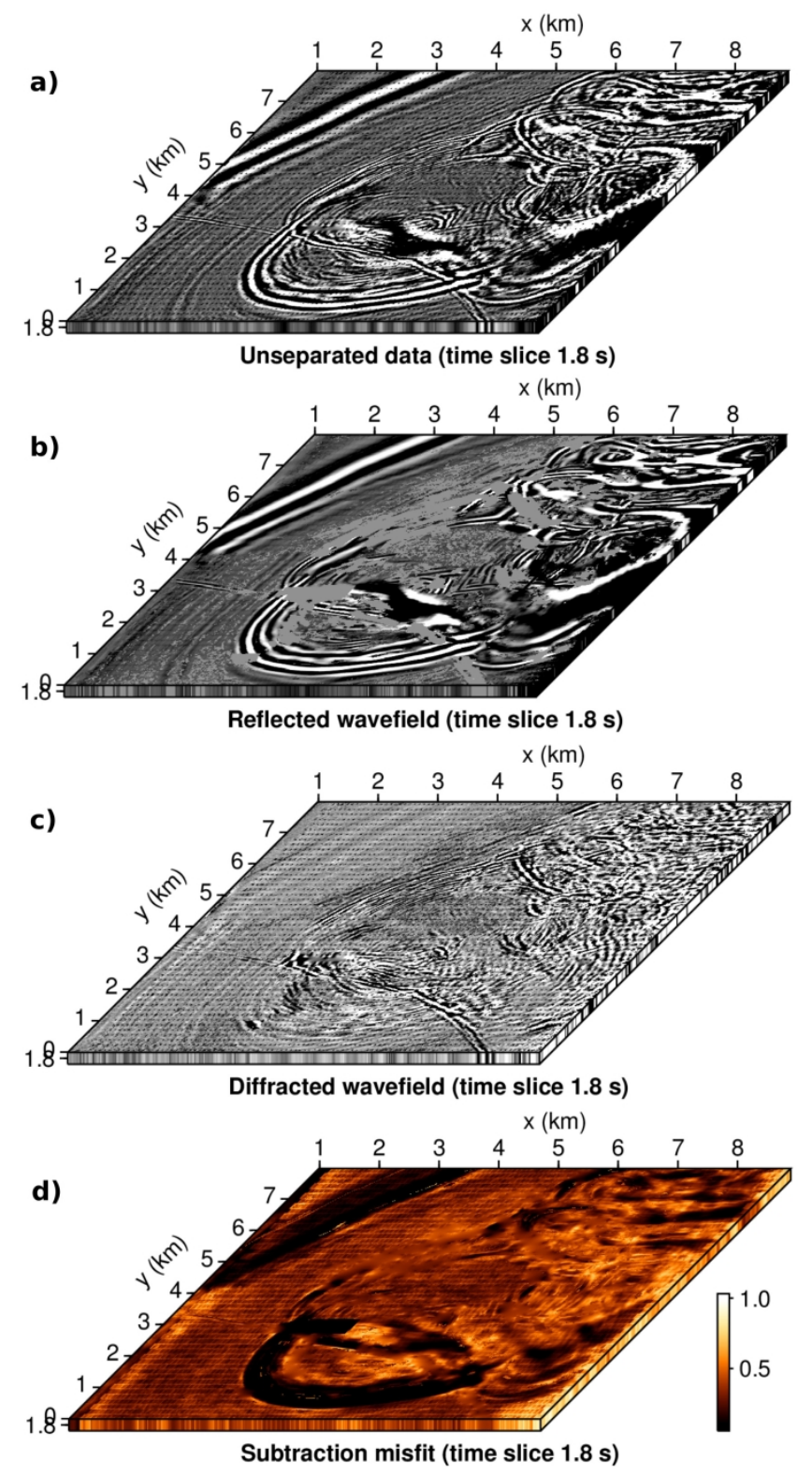

Figure 10. Illustration of the applicability of the coherent wavefield subtraction strategy for the 3D SEG/EAGE salt model seismic dataset. Shown are the $1.8 \mathrm{~s}$ time slices of (a) the input data, (b) the adapted reflection stack, (c) the coherent subtraction result, and (d) the normalized coherent subtraction misfit. Gaps in (b) indicate where dominant diffracted energy is detected and preserved through wavefront filters in the subtraction procedure.

$$
610 \times 1139 \mathrm{~mm}(600 \times 600 \mathrm{DPI})
$$


Figure 11. Line of a ground-penetrating radar (GPR) field dataset recorded by the US Geological Survey (USGS) on Long Beach Island in 2015 (Zaremba et al., 2016). Displayed are (a) the raw input data, (b) the separation result, and (c) the corresponding estimated misfit between subtracted data and input. Bright colors in (c) indicate where interference between reflection and diffraction occurs.

$$
610 \times 732 \mathrm{~mm}(600 \times 600 \mathrm{DPI})
$$




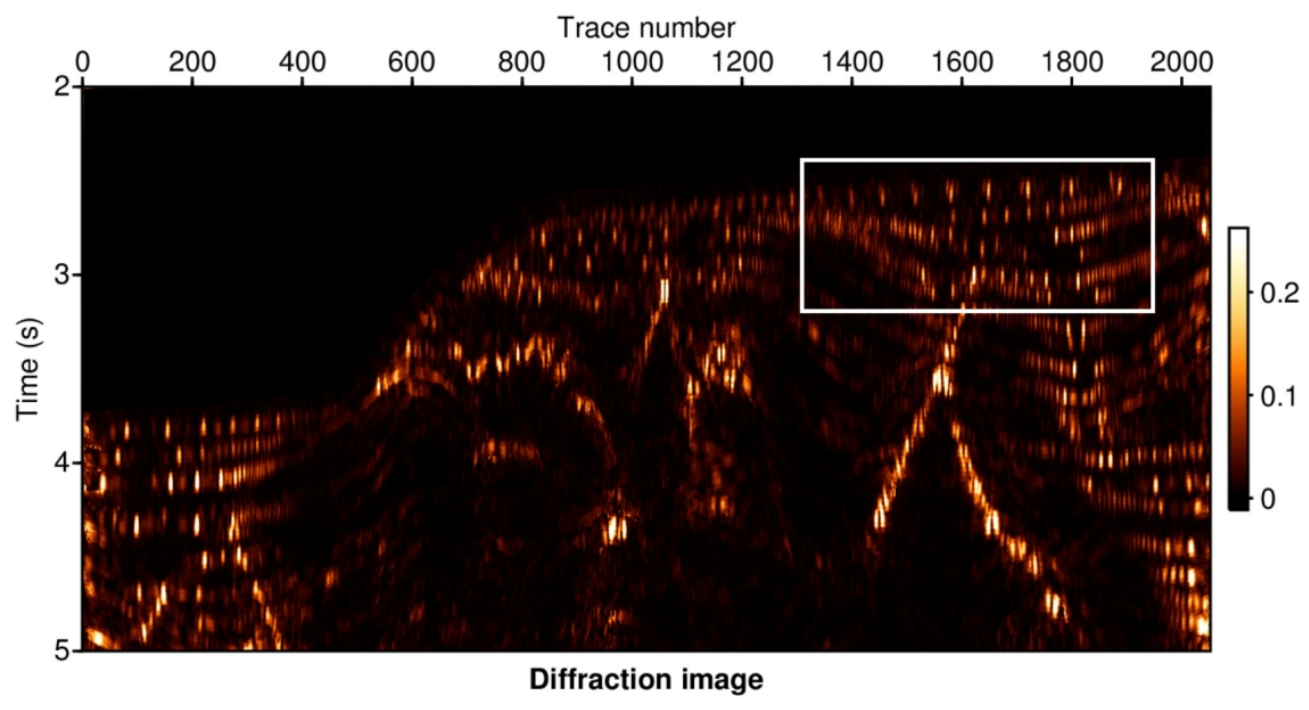

Figure 12. Diffraction (time) image of the Sigsbee 2A model gained through Kirchhoff-type focusing of the coherent wavefield subtraction result shown in Figure 6(b). In addition to dominant diffraction occuring at the top-of-salt, small-scale model discontinuities arising from the discretization of the model are detected.

$610 \times 325 \mathrm{~mm}(300 \times 300 \mathrm{DPI})$ 

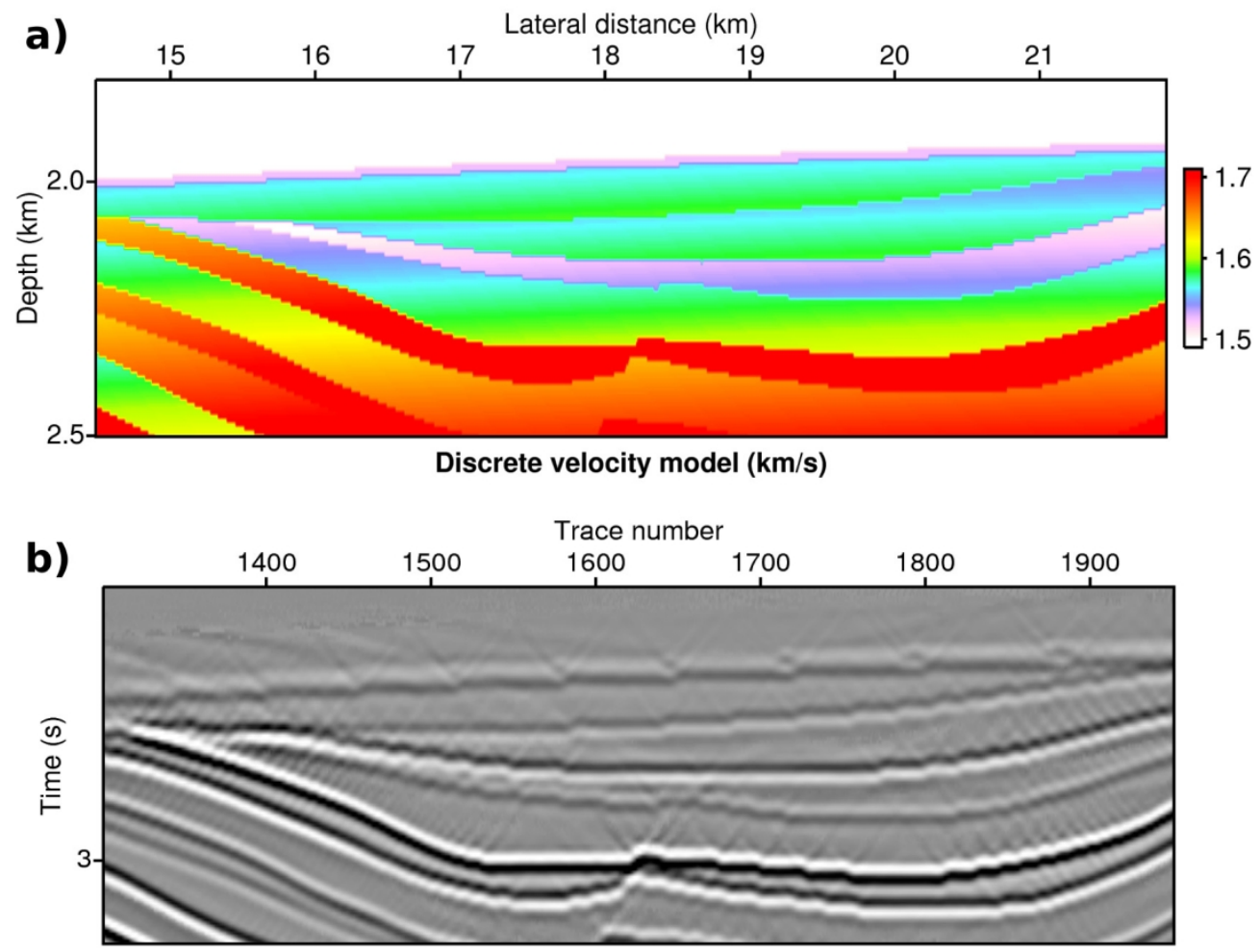

Conventional image

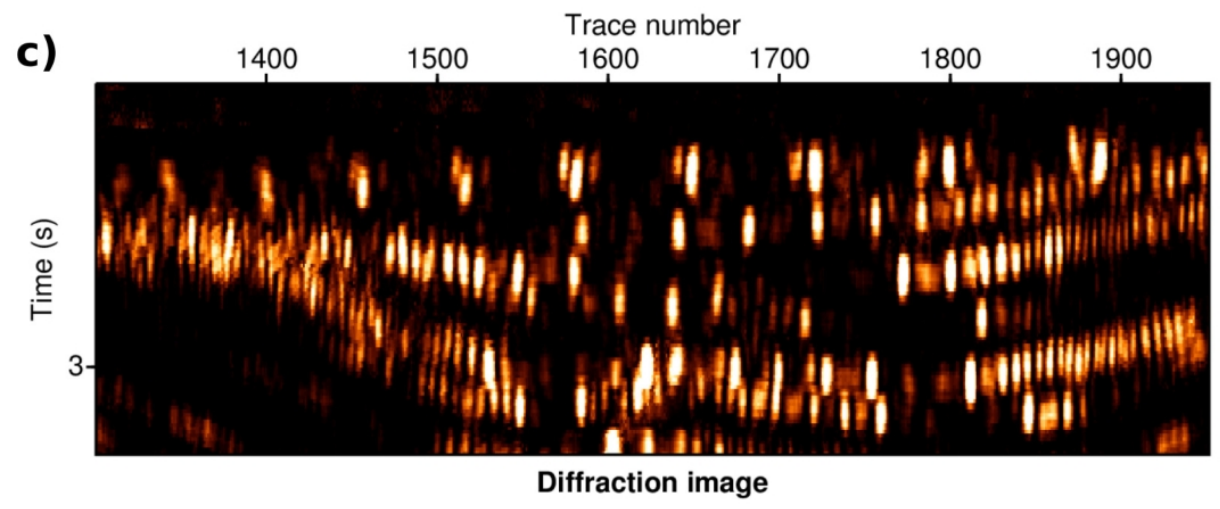

Figure 13. Closeup of the diffraction image (c) indicated by the frame in Figure 12, compared to the actual discrete depth model (a) and the conventional image resulting from the migration of the unseparated wavefield (b), in which reflection clearly dominates. The paired uppermost foci in (c) indicate that even the very thin shallow layer at approximately $2 \mathrm{~km}$ depth can be resolved.

$$
610 \times 733 \mathrm{~mm}(600 \times 600 \mathrm{DPI})
$$


DATA AND MATERIALS AVAILABILITY

Data associated with this research are available and can be obtained by contacting the corresponding author. 